\title{
Reduced-Order Modeling of Two-Dimensional Supersonic Flows with Applications to Scramjet Inlets
}

\author{
Derek J. Dalle,, Matt L. Fotia,,$*$ and James F. Driscoll $\ddagger$ \\ University of Michigan, Ann Arbor, Michigan 48109
}

DOI: $\underline{10.2514 / 1.46521}$

\begin{abstract}
Control-oriented models of hypersonic vehicle propulsion systems require a reduced-order model of the scramjet inlet that is accurate to within $10 \%$ but requires less than a few seconds of computational time. To achieve this goal, a reduced-order model is presented, which predicts the solution of a steady two-dimensional supersonic flow through an inlet or around any other two-dimensional geometry. The model assumes that the flow is supersonic everywhere except in boundary layers and the regions near blunted leading edges. Expansion fans are modeled as a sequence of discrete waves instead of a continuous pressure change. Of critical importance to the model is the ability to predict the results of multiple wave interactions rapidly. The rounded detached shock near a blunt leading edge is discretized and replaced with three linear shocks. Boundary layers are approximated by displacing the flow by an empirical estimate of the displacement thickness. A scramjet inlet is considered as an example application. The predicted results are compared to two-dimensional computational fluid dynamics solutions and experimental results.
\end{abstract}

\section{Nomenclature}

$=$ local sound speed, $\mathrm{m} / \mathrm{s}$

$=$ specific heat, $\mathrm{J} / \mathrm{kg} \cdot \mathrm{K}$

$=$ length normal to flow, $\mathrm{m}$

$=$ specific enthalpy, $\mathrm{J} / \mathrm{kg}$

$=$ length tangent to flow, $\mathrm{m}$

= Mach number

$=$ number of a given quantity

$=$ Prandtl number

$=$ pressure, $\mathrm{Pa}$

$=$ normalized gas constant, $\mathrm{J} / \mathrm{kg} \cdot \mathrm{K}$

$=8314.47 \mathrm{~J} / \mathrm{kmol} \cdot \mathrm{K}$

$=$ radius, $\mathrm{m}$

$=$ temperature, $\mathrm{K}$

$=$ velocity magnitude, $\mathrm{m} / \mathrm{s}$

$=$ molecular weight, $\mathrm{kg} / \mathrm{kmol}$

$=$ forward body-frame coordinate, $\mathrm{m}$

$=$ mass fraction

= vertical body-frame coordinate, $\mathrm{m}$

$=$ shock angle

$=$ ratio of specific heats

$=$ thickness of layer, $\mathrm{m}$

$=$ ratio

$=\ln p_{0} / p$

$=$ dynamic viscosity, $\mathrm{kg} / \mathrm{m} \cdot \mathrm{s}$

$=$ flowpath angle

$=v-\mu-\lambda+\pi / 2$

$=\sqrt{M^{2}-1}$

$=\sin ^{-1} 1 / M$, Mach angle

$=$ Prandtl-Meyer function

$=$ density, $\mathrm{kg} / \mathrm{m}^{3}$

= wave angle

$=$ flux of subscripted quantity

$=$ reference angle
Subscripts

$\begin{array}{lll}\mathrm{A}, \mathrm{B}, \ldots & =\text { region label } \\ \mathrm{a}, \mathrm{b}, \ldots & =\text { point label } \\ \mathrm{bs} & =\text { curved portion of bow shock } \\ \mathrm{cl} & =\text { property of inlet cowl } \\ e & =\text { value at edge of boundary layer } \\ \mathrm{ex} & =\text { expansion } \\ i & =\text { species index } \\ j & =\text { index of expansion discretization } \\ k & =\text { region index } \\ \mathrm{le} & =\text { leading edge } \\ p & =\text { constant pressure } \\ s & =\text { constant entropy } \\ \mathrm{sp} & =\text { pertaining to species } \\ w & =\text { wall value } \\ 0 & =\text { stagnation value } \\ 1 & =\text { index for inlet portion of flow } \\ 2 & =\text { index for inlet outflow } \\ \infty & =\text { freestream }\end{array}$

Superscripts

* $\quad=$ value at Mach number of 1

$+\quad=$ reference value for boundary layer

\section{Introduction}

$\mathbf{T}$ HE ability to estimate quickly the properties of a supersonic flow is critical for the design of a control-oriented model of a hypersonic vehicle. For example, a control algorithm must rapidly compute the thrust along a vehicle trajectory as the flight Mach number and angle of attack continuously change. The algorithm must also calculate the thrust for any perturbations to the design trajectory. Although simple tools such as Newtonian impact theory and piston theory can be used to estimate lift and drag, estimating the performance of a dual-mode scramjet requires accurate information about the properties of the fluid flow as it leaves the inlet and enters the isolator. The use of high-fidelity computational fluid dynamics (CFD) can be employed to determine the flow through the inlet accurately, but this solution requires too much computational time to be viable for control-oriented modeling. On the other hand, very simple models have been used by Bogar et al. [1], Bolender and

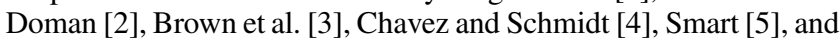
others to estimate the flow conditions in the inlet. These models ignore the effects of wave interactions and assume that the flow is uniform throughout the internal portion of the inlet. Although these
Received 28 July 2009; revision received 3 February 2010; accepted for publication 11 February 2010. Copyright (C) 2010 by Derek J. Dalle. Published by the American Institute of Aeronautics and Astronautics, Inc., Clearance Center, Inc., 222 Rosewood Drive, Danvers, MA 01923; include the code $0748-4658 / 10$ and $\$ 10.00$ in correspondence with the CCC.

*Graduate Research Assistant, Department of Aerospace Engineering. Member AIAA.

${ }^{\dagger}$ Professor, Department of Aerospace Engineering. Fellow AIAA. 
assumptions may be valid for a particular design under certain flight conditions, they become inaccurate when modeling flight at offdesign Mach numbers and angles of attack.

A satisfactory approach must require a relatively small amount of computational time and still yield a relatively accurate solution for the inlet flow. Instead of solving directly for the flow conditions at each point in the flow (as is done in CFD), the method presented here solves for the positions of the relevant waves, which separate regions in which the flow properties are uniform. The proposed method rapidly provides solutions for the locations of the shock waves and expansions using established two-dimensional supersonic theory. To make this possible in a digital computing environment, expansion fans are approximated as a number of discrete isentropic waves, and curved surfaces are modeled as a number of straight sections. After determining the locations at which two or more waves intersect, the program solves the interactions among the waves as a twodimensional Riemann problem. In many ways this is a generalized and automated version of the method of characteristics.

To account for blunted leading edges, the oblique shock is displaced a vertical distance from the leading edge to match the empirical shape of the detached shock given by Billig [6]. The wall boundary layer is approximated by displacing the flow by a distance equal to the boundary-layer displacement thickness, which is given by established empirical formulas.

An example scramjet inlet geometry, shown in Fig. 1, is used for validation throughout the paper. The coordinates of the vertices of this geometry are shown in Table 1 . The fourth shock is designed to turn the flow back to horizontal, and it exactly intersects the shoulder in the upper surface of the inlet. Therefore, there are no additional shocks downstream of this fourth shock. Under these flight conditions, the compression ratio of the inlet is $p_{2} / p_{\infty}=30.61$, and the pressure recovery factor is $p_{0,2} / p_{0, \infty}=0.6841$.

This geometry is selected to have exactly four shocks and no spillage for a flight Mach number of $M_{\infty}=8.0$ and an angle of attack of $\alpha=0$ assuming a sharp-nosed vehicle in an inviscid, calorically perfect (constant $c_{p}$ ) flow. The ideal shock waves for this condition are shown in light gray in Fig. 1. An example of the inviscid flow for a nonideal flight condition is shown in Fig. 2. When this inlet is operated at off-design Mach numbers, additional waves are formed, as shown in Fig. 2a. The expansion at the convex corner at station 1d, which is often called the shoulder, is modeled as two waves to highlight the discrete nature of the shock/expansion interaction in this model. The box in Fig. 2a illustrates the region shown in Fig. 2b, and the box in Fig. 2b illustrates a region in which a wave interaction problem occurs, which is solved as a Riemann problem. About 100 wave interactions occur in the inlet shown in Fig. 2 , which took $0.8 \mathrm{~s}$ to compute.

The most important utility of the proposed reduced-order model is the estimation of the inlet performance over a wide range of flow conditions. For this investigation, the freestream Mach numbers range from 6 to 12 and angles of attack from -2 to $+5 \mathrm{deg}$. Also of interest are the effects such as blunt noses, boundary layers, and varying specific heats. Although the proposed methods should yield accurate solutions to the inviscid flow problem, the greatly simplified viscous models are included in this report, mostly to show how they can be integrated with the rest of the solution algorithm.

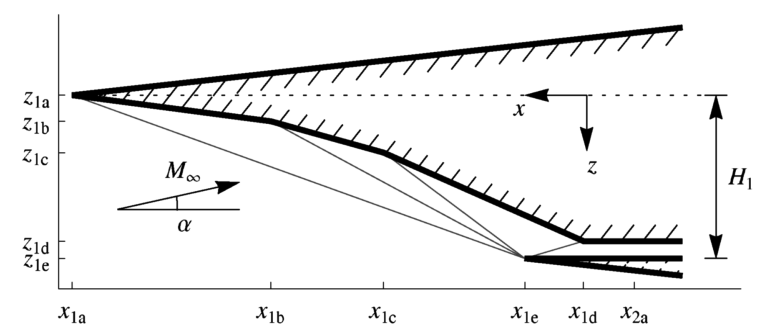

Fig. 1 Sketch of the coordinate system used for the sample inlet geometry, with the $x$ and $z$ axes shown on separate scales. The inlet height is $H_{1}=z_{1 \mathrm{e}}-z_{1 \mathrm{a}}$, and the height of the duct is $H_{2}=z_{1 \mathrm{e}}-z_{1 \mathrm{~d}}$.
Table 1 Scaled geometry of the vertices of the reference inlet with the physical scales set by $H_{1}=2.573 \mathrm{~m}$

\begin{tabular}{lcc}
\hline \hline & $\left(x_{1 k}-x_{1 \mathrm{a}}\right) / H_{1}$ & $\left(z_{1 k}-z_{1 \mathrm{a}}\right) / H_{1}$ \\
\hline $\mathrm{a}$ & 0 & 0 \\
$\mathrm{~b}$ & 2.5759 & 0.1592 \\
$\mathrm{c}$ & 4.0457 & 0.3512 \\
$\mathrm{~d}$ & 6.6416 & 0.8948 \\
$\mathrm{e}$ & 5.8824 & 1 \\
\hline \hline
\end{tabular}

Although the analysis of scramjet inlets was the primary motivation for developing this solution method, it can be applied to any two-dimensional geometry for which there are no subsonic regions except for boundary layers and small subsonic regions in the near vicinity of blunted noses. An example that demonstrates the flexibility of the program is shown in Fig. 3. In this solution, the four expansion fans generated at vertices of the airfoils are modeled as 20 discrete waves.

\section{High-Temperature Two-Dimensional Wave Model}

At the high static temperatures that occur in hypersonic flows, the assumption of a calorically perfect gas, which is defined as one for which $c_{p}$ is constant, becomes inaccurate. The well-established theory for two-dimensional shocks and expansions, therefore, is not applicable, and new equations for oblique shocks and expansion fans are required.

Although there is a well-established theory for two-dimensional shocks and expansions in a calorically perfect gas, the appropriate equations for a calorically imperfect gas are rarely presented in

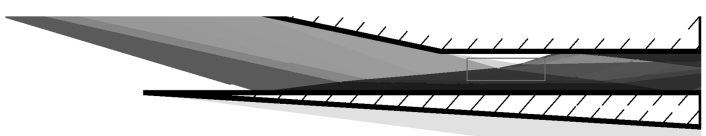

a) Inlet shoulder region

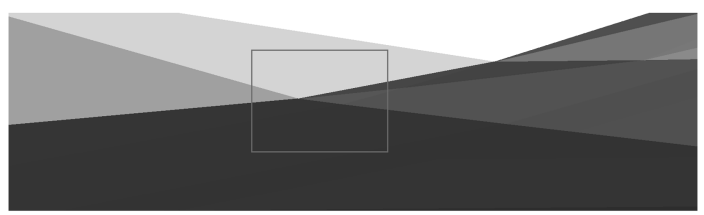

b) Interaction of shock and expansion

Fig. 2 Discretized inviscid flow through the inlet for a flight Mach number of $M_{\infty}=12.0$ and an angle of attack of $\alpha=0$. The darker shades represent regions of higher temperature; white represents freestream temperature, and black represents $T / T_{\infty}=8$. The expansion at the shoulder is modeled as two discrete waves.

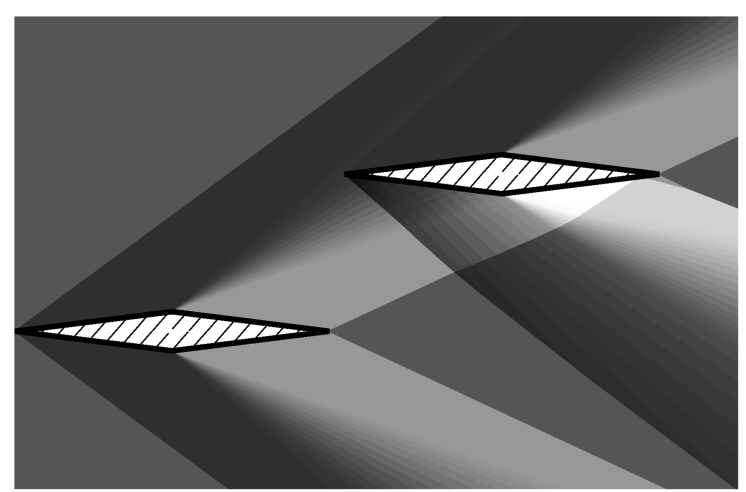

Fig. 3 Inviscid flow over two diamond airfoils at an angle of attack of $\alpha=0$ and a freestream Mach number of $M_{\infty}=2$, where the darker shades represent regions of higher pressure and white corresponds to the lowest pressure. 
textbooks. The Prandtl-Meyer expansion analysis presented here is similar to that of Emanuel [7], Ismail [8], and Zebbiche [9], whereas the oblique shock analysis is similar to that of Emanuel [7] and Tatum [10]. However, none of these authors considered wave interactions, which are the focus of the present work.

Two gas models are used with this proposed architecture for comparison. The first gas model is the calorically perfect gas model, in which the well-established two-dimensional shock/expansion theory is used. The second gas model assumes a calorically imperfect, nonreacting gas, in which specific heats vary with temperature, but the molecular composition of the gas is assumed to be constant. The equation of state $p=\rho R T$ remains valid. For air, significant amounts of oxygen begin to dissociate at static temperatures of $2500 \mathrm{~K}$. In the flows considered in this investigation, the flight Mach numbers ranged from 6.0 to 12.0 with freestream temperatures between 200 and $300 \mathrm{~K}$. In these flows, gas dissociation does not play a major role, and the nonreacting gas model is accurate. However, for higher-temperature flows, a gas model that accounts for chemical reactions would be needed.

The following subsections describe the calorically imperfect gas model that was used and how it was applied to model waves and wave interactions. In addition, a method for splitting a continuous twodimensional expansion wave into discrete waves is discussed. The discrete expansion waves must satisfy all of the conservation laws that are satisfied by the continuous expansion.

\section{A. Calorically Imperfect Gas Model}

For a real gas with $n_{\text {sp }}$ species, the specific enthalpy as a function of temperature is

$$
h=\sum_{i=1}^{n_{\mathrm{sp}}} h_{i} Y_{i}
$$

where $h_{i}$ is the specific enthalpy of species $i$, which is itself a nonconstant function of $T$. For a nonreacting (frozen) gas, the specific heat at constant pressure is

$$
c_{p}=\sum_{i=1}^{n_{\mathrm{sp}}} c_{p, i} Y_{i}
$$

where $c_{p, i}=\partial h_{i} / \partial T$ is the specific heat of species $i$. The ratio of specific heats is

$$
\gamma=\frac{c_{p}}{c_{p}-R}
$$

where

$$
R=\mathcal{R} \sum_{i=1}^{n_{\mathrm{sp}}} \frac{Y_{i}}{W_{i}}
$$

is the normalized gas constant for the mixture.

The square of the sound speed is equal to the derivative of the pressure with respect to the density under conditions of constant entropy; $a^{2}=(\partial p / \partial \rho)_{s}$. For a nonreacting ideal gas, the result is

$$
a^{2}=(\gamma p / \rho)=\gamma R T
$$

For a gas with a static temperature $T$ and local velocity $u$, the stagnation enthalpy is

$$
h_{0}=h(T)+\frac{1}{2} u^{2}
$$

and the stagnation temperature is then given by solving $h_{0}=h\left(T_{0}\right)$. The stagnation pressure, which is the pressure that would be measured if the flow is isentropically brought to rest, is

$$
\frac{p_{0}}{p}=\exp \left(\int_{T}^{T_{0}} \frac{\gamma}{\gamma-1} \frac{\mathrm{d} T}{T}\right)
$$

Then, for states $\mathrm{A}$ and $\mathrm{B}$ with the same stagnation pressure, the pressure ratio is

$$
\left(p_{\mathrm{B}} / p_{\mathrm{A}}\right)=\exp \left(\zeta\left(T_{\mathrm{A}}\right)-\zeta\left(T_{\mathrm{B}}\right)\right)
$$

where $\zeta\left(T_{0}\right)=0$, and

$$
\frac{\mathrm{d} \zeta}{\mathrm{d} T}=\frac{\gamma}{1-\gamma} \frac{1}{T}=-\frac{c_{p}}{R T}
$$

Because $h_{0}$ is constant, $\zeta$ can be calculated beforehand and used at any point in the flow.

\section{B. Prandtl-Meyer Expansions}

Prandtl-Meyer theory is used to predict the flow properties of an expanding supersonic flow. To account for the varying $c_{p}$ of a calorically imperfect gas, it is necessary to rewrite the equations for the Prandtl-Meyer function. In addition to determining the conditions downstream, Prandtl-Meyer theory can be used to predict the conditions along the characteristics within the expansion fan. The geometry of this situation is illustrated in Fig. 4 .

Assuming $\theta_{\mathrm{B}}<\theta_{\mathrm{A}}$, the conditions downstream of a twodimensional expansion can be found using the equation

$$
\theta_{\mathrm{B}}-\theta_{\mathrm{A}}=\int_{M_{\mathrm{B}}}^{M_{\mathrm{A}}} \sqrt{M^{2}-1} \frac{\mathrm{d} u}{u}
$$

This expression comes from a geometric argument [11] that is independent of the relationship between $h$ and $T$. This can also be written as a characteristic equation as

$$
v_{\mathrm{B}}+\theta_{\mathrm{B}}=v_{\mathrm{A}}+\theta_{\mathrm{A}}
$$

where $v$ is the Prandtl-Meyer angle. Because $u=M a$, one can write

$$
\frac{\mathrm{d} u}{u}=\frac{\mathrm{d} M}{M}+\frac{\mathrm{d} a}{a}
$$

for any gas model. Define three new expressions of

$$
\begin{gathered}
\kappa=\int_{1}^{M} \sqrt{M^{\prime 2}-1} \frac{\mathrm{d} a}{a} \\
\lambda=\sqrt{M^{2}-1}
\end{gathered}
$$

$$
\mu=\sin ^{-1}(1 / M)=\tan ^{-1}(1 / \lambda)
$$

Then Eqs. (10-15) yield the expression

$$
v=\mu+\lambda+\kappa-\pi / 2
$$

for the Prandtl-Meyer angle.

In general, the differential element $\mathrm{d} a$ cannot be written as a simple function of $M$ and $\gamma$. Instead, the strategy is to write both $a$ and $M$ as a function of $T$. The derivative of the sound speed with respect to temperature is

$$
\frac{\mathrm{d} a}{\mathrm{~d} T}=\frac{1}{2}\left(\frac{1}{\gamma} \frac{\mathrm{d} \gamma}{\mathrm{d} T}+\frac{1}{T}\right)
$$

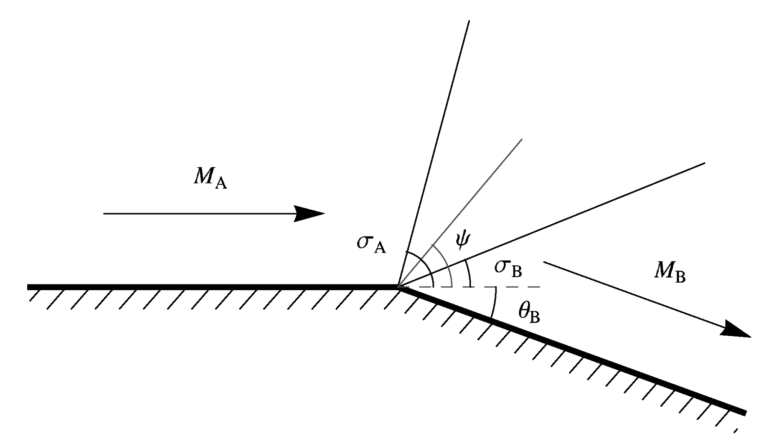

Fig. 4 Illustration of Prandtl-Meyer expansion wave. 
and the local Mach number is

$$
M^{2}=2\left(h_{0}-h\right) / a^{2}
$$

Although Eq. (13) appears at first to be a daunting expression, the situation is not so difficult if the flow is adiabatic.

Because $h_{0}$ is constant, $\kappa$ can be calculated beforehand and used for all the expansions in a flow. Taking the derivative of Eq. (13) gives the differential equation

$$
\frac{\mathrm{d} \kappa}{\mathrm{d} T}=\frac{1}{2}\left(\frac{1}{\gamma} \frac{\mathrm{d} \gamma}{\mathrm{d} T}+\frac{1}{T}\right) \sqrt{M^{2}-1}
$$

with the initial condition $\kappa\left(T^{*}\right)=0$. The critical temperature $T^{*}$ is the temperature of the flow when isentropically decelerated to a Mach number of 1 . Once the downstream temperature has been calculated, the corresponding flow velocity is

$$
u_{\mathrm{B}}=\sqrt{2\left(h_{0}-h\left(T_{\mathrm{B}}\right)\right)}
$$

The downstream pressure is given by Eq. ( $\underline{8})$. For a calorically perfect gas, a useful result is

$$
\kappa=-\lambda+\sqrt{\frac{\gamma+1}{\gamma-1}} \tan ^{-1} \sqrt{\frac{\gamma-1}{\gamma+1} \lambda^{2}}
$$

which gives the traditional value for the Prandtl-Meyer function.

The angle of the first characteristic is $\sigma_{\mathrm{A}}=\mu_{\mathrm{A}}$, and the last characteristic has an angle of $\sigma_{\mathrm{B}}=\mu_{\mathrm{B}}+\theta_{\mathrm{B}}$. However, this does not resolve the flow between the first and last characteristics. To find the Mach number along a characteristic for which the angle with the freestream velocity is $\psi$, the characteristic equation is

$$
v_{\mathrm{A}}=v(\psi)+\theta(\psi)
$$

Because $\psi$ is a wave angle, it is the sum of the local flowpath angle and the characteristic angle. Combining this fact with Eq. (22) yields the expression

$$
\psi=v_{\mathrm{A}}+\mu(\psi)-v(\psi)
$$

For a calorically perfect gas, this equation has an explicit solution:

$$
M=\sqrt{1+\frac{\gamma+1}{\gamma-1} \tan ^{2}\left(\sqrt{\frac{\gamma-1}{\gamma+1}}\left(v_{\mathrm{A}}-\psi+\frac{\pi}{2}\right)\right)}
$$

The resolution within the expansion wave, given in Eq. (23), provides a method to discretize the wave. By selecting a set of angles, $\psi_{1}, \ldots, \psi_{n_{\mathrm{ex}}}$, for evaluation and wave angles, the wave can be split into a set of regions within which the properties such as Mach number are constant. The regions are separated by discrete waves, which have angles of $\sigma_{1}, \ldots, \sigma_{n_{\mathrm{ex}}}$. Then the approximation is

$$
\tilde{M}(\psi)=M\left(\psi_{j}\right)
$$

where $\sigma_{j} \leq \psi \leq \sigma_{j+1}$.

The current program selects values of $\psi_{j}$ according to the rules of Gaussian quadrature, but any distribution of angles between $\sigma_{\mathrm{B}}$ and $\sigma_{\mathrm{A}}$ is valid. Once the evaluation angles, $\psi_{1}, \ldots, \psi_{n_{\mathrm{ex}}}$, have been selected, the intermediate temperatures, $T_{1}, \ldots, T_{n_{\mathrm{ex}}}$, are calculated using Eq. (23).

The wave angles are selected so that mass flux through the expansion wave is conserved. This gives the result

$$
\sin \sigma_{j}=\frac{\sin \theta_{j-1}-\varepsilon_{j} \sin \theta_{j}}{\sqrt{1+\varepsilon_{j}^{2}-2 \varepsilon_{j} \cos \left(\theta_{j}-\theta_{j-1}\right)}}
$$

where $\varepsilon_{j}=\rho_{j} u_{j} / \rho_{j-1} u_{j-1}$ is the ratio of nominal mass fluxes before and after the $j$ th discrete wave.

\section{Oblique Shocks}

It is necessary to rewrite the oblique shock equations to account for the varying $c_{p}$ of a calorically imperfect gas. The present model is valid for any gas that obeys the equation of state $p=\rho R T$ and has a known relationship between $h$ and $T$. The geometry for this problem is shown in Fig. 5.

The postshock flow must be tangent to the surface, so conservation of mass implies

$$
\rho_{\mathrm{A}} u_{\mathrm{A}} H_{\mathrm{A}}=\rho_{\mathrm{B}} u_{\mathrm{B}} H_{\mathrm{B}}
$$

The characteristic length scales $H_{\mathrm{A}}$ and $H_{\mathrm{B}}$ are determined by considering any point $b$ on the shock in Fig. $\underline{5}$. Then

$$
H_{\mathrm{B}}=L_{\mathrm{A}} \tan \left(\beta-\theta_{\mathrm{B}}\right)
$$

and

$$
H_{\mathrm{A}}=L_{\mathrm{A}}\left(\sin \theta_{\mathrm{B}}+\tan \left(\beta-\theta_{\mathrm{B}}\right) \cos \theta_{\mathrm{B}}\right)
$$

Conservation of momentum in the direction tangent to the shock states that the component of the velocity tangent to the shock is the same upstream and downstream of the shock. Then

$$
u_{\mathrm{A}} \cos \beta=u_{\mathrm{B}} \cos \left(\beta-\theta_{\mathrm{B}}\right)
$$

Define a compression ratio $\varepsilon=\rho_{\mathrm{A}} / \rho_{\mathrm{B}}$. Because the postshock density will always be greater than the preshock density, $\varepsilon$ varies between 0 and 1. Equation (27) then has the solution

$$
\tan \left(\beta-\theta_{\mathrm{B}}\right)=\frac{1-\varepsilon}{2 \tan \theta_{\mathrm{B}}}-\sqrt{\left(\frac{1-\varepsilon}{2 \tan \theta_{\mathrm{B}}}\right)^{2}-\varepsilon}
$$

Conservation of momentum in the direction normal to the shock states that the sum of the static and dynamic pressures normal to the shock is unaffected by the presence of the shock. Thus,

$$
\left(p_{B} / p_{A}\right)=1+\gamma M_{\mathrm{A}}^{2}(1-\varepsilon) \sin ^{2} \beta
$$

Finally, conservation of energy requires that the stagnation enthalpy is unaffected by the shock. Therefore,

$$
h_{\mathrm{B}}=h_{\mathrm{A}}+\frac{1}{2} u_{\mathrm{A}}^{2}\left(1-\varepsilon^{2}\right) \sin ^{2} \beta
$$

Equations (31-33) represent three independent equations for the three unknowns, $h_{\mathrm{B}}, p_{\mathrm{B}}$, and $u_{\mathrm{B}}$, downstream of the shock.

For a calorically perfect gas, $T_{\mathrm{B}}=h_{\mathrm{B}} / c_{p}$, and the equations can be reduced to functions of $\beta, \theta_{\mathrm{B}}$, and $M_{\mathrm{A}}$ only. For another gas model, $h_{\mathrm{B}}=h\left(T_{\mathrm{B}}\right)$ must be solved iteratively. As with the calorically perfect gas, the compression ratio is not a function of the preshock pressure. However, the initial absolute temperature now plays a significant role.

\section{Two-Dimensional Riemann Problem}

Consider the case of two interacting waves, which are shown on the left side of Fig. 6. The important parameters are the gas properties in regions $A$ and $\bar{D}$. The flow pattern on the right side of Fig. 6 is called the Riemann problem. It describes the situation in steady twodimensional compressible flow in which two uniform regions are in

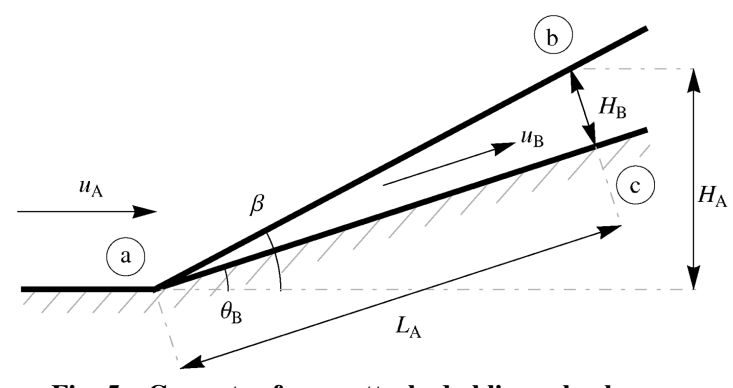

Fig. 5 Geometry for an attached oblique shock wave. 


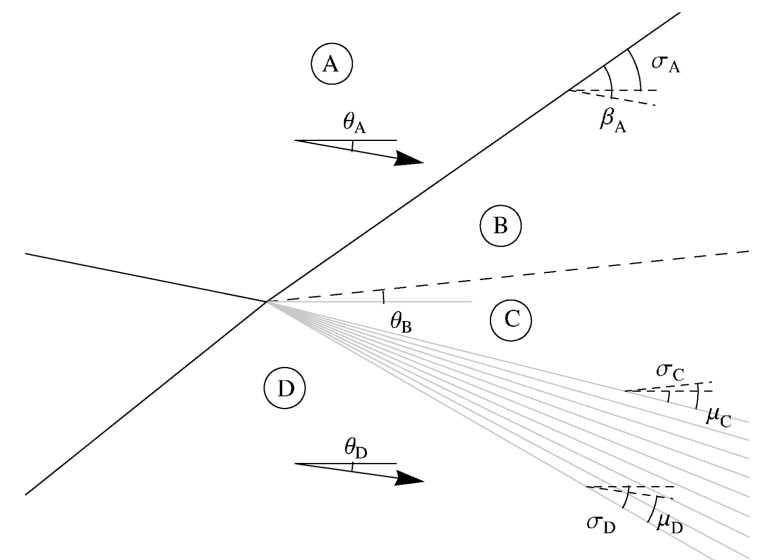

Fig. 6 Geometry for a wave interaction with a shock and an expansion. The region of flow in the graphic is the region of the box in Fig. 2 . In the general Riemann problem, both waves could be either a shock or an expansion. The combination of waves here indicates $p_{\mathrm{D}}>p_{\mathrm{A}}$.

contact with each other. At the point of interaction, there are two inconsistent flow conditions. To rectify this situation, the gas in both regions must pass through a wave, as shown in Fig. 6 .

The physical solution must have the property that the regions that are not separated by a shock or expansion must have equal pressures and flow in the same direction. Thus,

$$
p_{\mathrm{B}}=p_{\mathrm{C}} \quad \text { and } \quad \theta_{\mathrm{B}}=\theta_{\mathrm{C}}
$$

A simple, though inefficient, solution method is to combine the results of Secs. II.B and II.C into a single pressure-deflection function:

$$
p_{\mathrm{B}}=p\left(\mathrm{~A}, \theta_{\mathrm{B}}-\theta_{\mathrm{A}}\right)
$$

which takes as input all information about state $\mathrm{A}$ and a deflection angle $\theta_{\mathrm{B}}-\theta_{\mathrm{A}}$, and returns a postwave pressure as output. If $\theta_{\mathrm{B}}<\theta_{\mathrm{A}}$, the expansion relations of Sec. II.B are used, whereas if $\theta_{\mathrm{B}}>\theta_{\mathrm{A}}$, the shock relations of Sec. II.C are used. The same function can be used to determine $p_{\mathrm{C}}$ using the formula

$$
p_{\mathrm{C}}=p\left(\mathrm{D}, \theta_{\mathrm{D}}-\theta_{\mathrm{C}}\right)
$$

Opposite signs are used for the lower shock because the wave separating regions $\mathrm{C}$ and $\mathrm{D}$ is of the opposite family as the wave separating regions $\mathrm{A}$ and $\mathrm{B}$. Combining this pressure-deflection function with the constraints of Eq. (34) gives an equation

$$
p\left(\mathrm{~A}, \theta_{\mathrm{B}}-\theta_{\mathrm{A}}\right)=p\left(\mathrm{D}, \theta_{\mathrm{D}}-\theta_{\mathrm{B}}\right)
$$

which is satisfied only by the correct value of $\theta_{\mathrm{B}}$.

To reduce the number of waves present in a flow solution, it is advantageous to ignore waves that cause a negligible change in conditions. For example, if $T_{\mathrm{B}} / T_{\mathrm{A}}$ is very close to unity, the wave separating regions $\mathrm{A}$ and $\mathrm{B}$ plays a very minor role. In the proposed method, therefore, any of the three waves resulting from a wave interaction is ignored if the temperature jump across it is below a certain tolerance.

\section{Proposed Method of Supersonic Flow Discretization}

The proposed reduced-order model is primarily concerned with the lines that separate regions of the flow that are considered to have uniform properties. These lines can be shock waves, discretized expansion waves, or solid surfaces, and they are referred to as paths in the following description. As a result, the output of the program is a list of polygons and corresponding lists that give the pressure, density, etc., in each polygon. This is very similar to the output of a two-dimensional finite volume code, only the grid is very coarse in most of the flow. However, because the polygons are assembled during the flow computation, there is no need to assemble a grid a priori.

In addition to the wave theory of Sec. II, models are constructed for regions of the flow in which the local Mach number is less than one. Because information can travel upstream in these regions, they are somewhat incompatible with the rest of the solution. The simple models presented here provide an architecture that allows them to be integrated with the otherwise supersonic flow.

\section{A. Description of the Proposed Algorithm}

The input to the program consists of two parts. The first part determines the geometry of the flow, and it requires a list of polygons that specify the solid surfaces in the flow. For example the reference inlet shown in Fig. 1 has two input polygons: a list of six vertices for the inlet ramp section and a list of three vertices for the cowl. The second part of the input is the initial conditions. These are the conditions upstream of the input polygons. For example, in Fig. 3, the initial conditions specify the flow along the left edge of the image.

Once the flow geometry and initial conditions have been specified, the program proceeds by marching downstream and searching for wave interactions or vertices of the input geometry. To accomplish this, the program begins its analysis at the farthest upstream $x$ coordinate, $x_{\max }$, and proceeds downstream toward the right-hand $x$ coordinate, $x_{\min }$. As the program proceeds downstream, it tracks the positions of all the straight lines, or paths, in the flow. These paths include waves, the surfaces of the input geometry, and the boundaries of the flow domain. Within the program each of these straight lines is tracked as a path that consists of a point and a propagation angle. The program keeps track of all of the paths that intersect the vertical line corresponding to the current $x$ coordinate.

With the set of paths at a given $x$ coordinate given, a list of possible intersection points can be determined. Suppose the current $x$ coordinate is $x_{k}$, and the paths are listed by ascending $z$ coordinates. Then two paths with coordinates $z_{j}$ and $z_{j+1}$ have a downstream intersection point of

$$
x_{k, j}=x_{k}+\frac{z_{j+1}-z_{j}}{\tan \sigma_{j+1}-\tan \sigma_{j}}
$$

provided that $\sigma_{j}<\sigma_{j+1}$. Then the $x$ coordinate of the next interaction point, $x_{k+1}$, is the minimum of all the $x_{k, j}$ and all of the vertices of the input polygons that are downstream of $x_{k}$.

Once an interaction point is found, a three-step process takes place. First, the nature of the interaction is determined, and the local flow problem is solved. This consists of determining the state (pressure, flowpath angle, etc.) above and below the interaction point. If one of the paths is a surface boundary, the downstream conditions are determined by either a shock or an expansion. Otherwise, the solution is determined by a Riemann problem as described in Sec. II.D. The second step is to add the coordinates of the interaction point to the polygons of the interacting regions. Finally, the list of waves and their propagation angles at the current $x$ coordinate are updated so that the coordinates of the next interaction point can be determined.

\section{B. Downstream Averaging Model}

At the downstream boundary of the reference inlet geometry, it is useful to define the spatially averaged gas properties, which can be input into a one-dimensional isolator or combustor model. With the proposed inlet model, gas properties are not uniform at the downstream boundary of the inlet because of the possible presence of waves separating regions of different gas properties. The proposed method to determine spatially averaged properties requires that the total fluxes of mass, momentum, and stagnation enthalpy are constant through the $x=x_{2 \mathrm{a}}$ plane. The geometry of this scheme is shown in Fig. 7.

The mass flux into the averaging plane is

$$
\Phi_{\rho}=\rho_{2} u_{2} H_{2}
$$




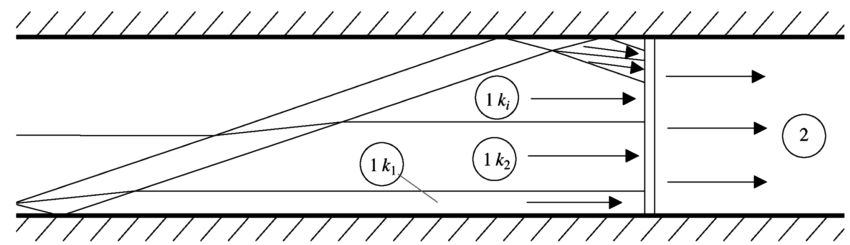

Fig. 7 Geometry of the averaging plane at $x=x_{2 \mathrm{a}}$ for the duct portion of the inlet. The black lines represent waves from a solution to the flow through the reference inlet. Region $1 k_{1}$ is the lowest region that has a boundary along the averaging plane, region $1 k_{2}$ is the region above that, etc. In this example, there are $n_{2}=6$ regions that contact the averaging plane.

$$
\Phi_{\rho}=\sum_{i=1}^{n_{2}} \rho_{1 k_{i}} u_{1 k_{i}} H_{1 k_{i}} \cos \theta_{1 k_{i}}
$$

where $H_{1 k_{i}}$ is the height of region $1 k_{i}$ at its downstream edge. Similarly, the momentum flux out of the inlet is

$$
\Phi_{u}=\rho_{2} u_{2}^{2} H_{2}
$$

whereas the momentum flux into the averaging plane is

$$
\Phi_{u}=\sum_{i=1}^{n_{2}} \rho_{1 k_{i}} u_{1 k_{i}}^{2} H_{1 k_{i}} \cos ^{2} \theta_{1 k_{i}}
$$

Finally, the flux of stagnation enthalpy is

$$
\Phi_{h}=\rho_{2} u_{2} H_{2} h_{0,2}
$$

and the flux into the plane is

$$
\Phi_{h}=\sum_{i=1}^{n_{2}} \rho_{1 k_{i}} u_{1 k_{i}} h_{0,1 k_{i}} H_{1 k_{i}} \cos \theta_{1 k_{i}}
$$

Once the fluxes into the averaging plane have been calculated, the state variables for the flow out of the inlet can be determined. The density is $u_{2}=\Phi_{u} / \Phi_{\rho}$, the stagnation enthalpy is $h_{0,2}=\Phi_{h} / \Phi_{\rho}$, and the density is

$$
\rho_{2}=\frac{\Phi_{\rho}}{u_{2} H_{2}}
$$

Then

$$
h_{2}=h_{0,2}-\frac{1}{2} u_{2}^{2}
$$

and the pressure can be calculated from the equation of state.

\section{Blunted Leading Edges}

Because a real leading edge will not be infinitely sharp, a model is needed to account for the curved bow shock around the leading edge. The geometry of this problem is shown in Fig. $\underline{8}$. To minimize computational time, the model must be relatively simple. Billig reports [6] that the shock shape is approximately

$$
\left(\frac{x_{\mathrm{b}}-x_{\mathrm{bs}}}{r_{\mathrm{le}}}+1+\frac{\delta_{\mathrm{bs}}}{r_{\mathrm{le}}}\right) \tan ^{2} \sigma=\frac{r_{\mathrm{bs}}}{r_{\mathrm{le}}}\left(\sqrt{1+\left(\frac{z_{\mathrm{b}}-z_{\mathrm{bs}}}{r_{\mathrm{bs}}} \tan \sigma\right)^{2}}-1\right)
$$

where $\left(x_{\mathrm{bs}}, z_{\mathrm{bs}}\right)$ are the coordinates of a point on the bow shock, $r_{\mathrm{le}}$ is the nose radius, $r_{\mathrm{bs}}$ is the radius of curvature of the shock along the stagnation streamline, $\delta_{\mathrm{bs}}$ is the standoff distance between the shock and the stagnation point, and $\sigma$ is the angle of the oblique shock that would be generated by the sharp nose with the same deflection angle. This expression results from the assumption that the shock shape should be a hyperbola and that, far away from the leading edge, the shock should be oblique with the same shock angle as a sharp leading edge would generate. A suggested correlation for the shock radius of curvature is [6]

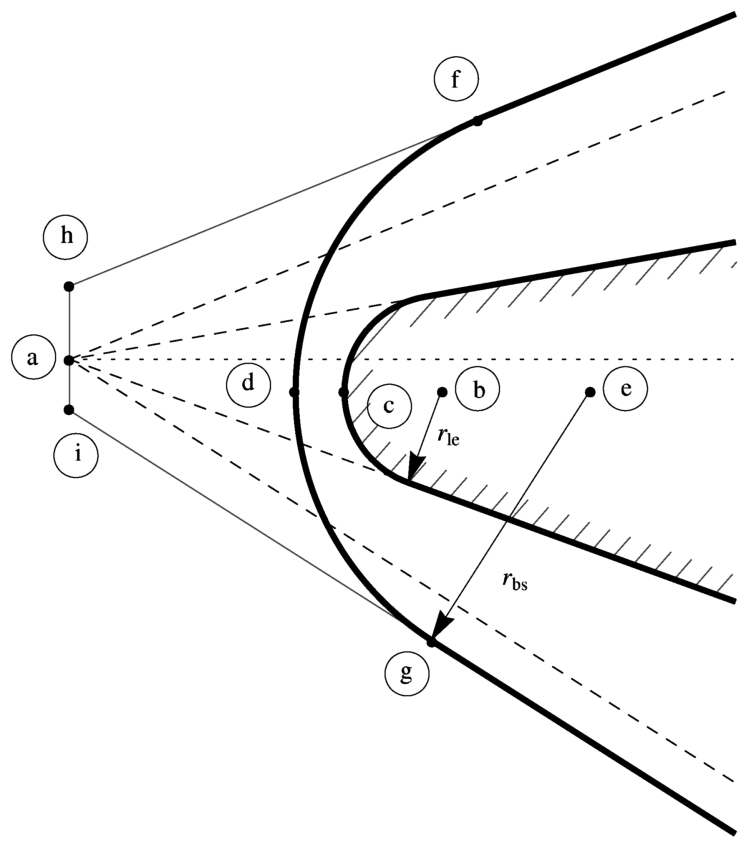

a) Comparison of sharp and blunted leading edges. Points are labeled to clarify the calculations in Sec. III.C

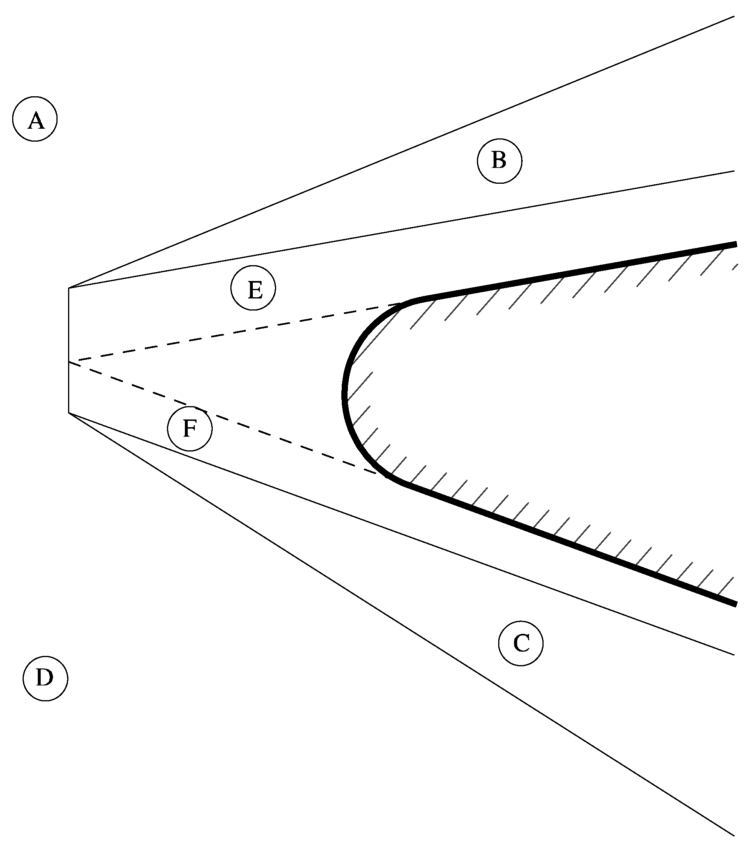

b) Regions associated with model for blunt leading edge. Regions $\mathrm{E}$ and $\mathrm{F}$ have a higher entropy than regions $\mathrm{B}$ and $\mathrm{C}$, respectively, as a result of passing through the bow shock between points $f$ and $g$

Fig. 8 Graphical description of model for blunt leading edges. The dashed lines represent the sharp leading edge and the oblique shocks attached to it, and the hashed bold line represents the blunted leading edge. The thick bold curve is the bow shock around the blunted leading edge, and the gray line connecting points $f, h, i$, and $g$ is the path of the shock used in the model.

$$
\frac{r_{\mathrm{bs}}}{r_{\mathrm{le}}}=1.386 \exp \left(\frac{1.8}{\left(M_{\mathrm{A}}-1\right)^{0.75}}\right)
$$

and the standoff distance is

$$
\frac{\delta_{\mathrm{bs}}}{r_{\mathrm{le}}}=0.386 \exp \left(\frac{4.67}{M_{\mathrm{A}}^{2}}\right)
$$


These results are obtained from experimental results in which the deflection angles are large, and there is evidence that they are not accurate when the deflection angles are small [3]. When $\left(z_{\mathrm{bs}}-\right.$ $\left.z_{\mathrm{b}}\right) / r_{\text {le }}$ is large, Eq. (47) asymptotically converges to an oblique shock that connects points $h$ and $f$ or points $i$ and $g$ in Fig. $\underline{\text { a }}$ depending on which value of $\sigma$ is used.

Consider a general case in which the leading edge is situated so that the preshock state above the leading edge is state A and the preshock state below the leading edge is D, as marked in Fig. $\underline{8}$. In most cases, states A and D are identical. In the case of a sharp nose, the upper downstream state, $\mathrm{B}$, is calculated using the standard oblique shock relations with a deflection angle $\theta_{\mathrm{B}}-\theta_{\mathrm{A}}$. In the bluntleading-edge model, states $\mathrm{B}$ and $\mathrm{C}$ are calculated in the same way as in the sharp-leading-edge model, but the oblique shock is displaced some distance in the $z$ direction. This creates extra regions, $\mathrm{E}$ and $\mathrm{F}$, which must be calculated in some other manner.

Assuming that $\theta_{\mathrm{B}}$ and $-\theta_{\mathrm{C}}$ are not necessarily the same angle, the center of the circle that forms the blunt leading edge is located at

$$
\frac{x_{\mathrm{a}}-x_{\mathrm{b}}}{r_{\mathrm{le}}}=\frac{\cos \frac{1}{2}\left(\theta_{\mathrm{B}}+\theta_{\mathrm{C}}\right)}{\sin \frac{1}{2}\left(\theta_{\mathrm{B}}-\theta_{\mathrm{C}}\right)}
$$

and

$$
\frac{z_{\mathrm{a}}-z_{\mathrm{b}}}{r_{\mathrm{le}}}=\frac{\sin \frac{1}{2}\left(\theta_{\mathrm{B}}+\theta_{\mathrm{C}}\right)}{\sin \frac{1}{2}\left(\theta_{\mathrm{B}}-\theta_{\mathrm{C}}\right)}
$$

Then the $z$ coordinate of point $h$ is

$$
\frac{z_{\mathrm{h}}-z_{\mathrm{b}}}{r_{\mathrm{le}}} \cot \sigma_{\mathrm{B}}=\frac{x_{\mathrm{a}}-x_{\mathrm{b}}+\delta_{\mathrm{bs}}}{r_{\mathrm{le}}}-1+\frac{r_{\mathrm{bs}}}{r_{\mathrm{le}}} \cot ^{2} \sigma_{\mathrm{B}}
$$

and the $z$ coordinate of point $i$ is found using the same formula, only replacing $\sigma_{\mathrm{B}}$ with $\sigma_{\mathrm{C}}$. The vertical displacement distances are $H_{\mathrm{E}}=$ $z_{\mathrm{h}}-z_{\mathrm{a}}$ and $H_{\mathrm{F}}=z_{\mathrm{i}}-z_{\mathrm{a}}$.

The remaining task is to estimate the conditions in regions $\mathrm{E}$ and $\mathrm{F}$. These regions will not be uniform in an actual flow because fluid passes through different points on a curved shock. The streamline passing through point $\mathrm{f}$ in Fig. 8a has the same postshock conditions as region $\mathrm{B}$, whereas the streamline that intersects the shock at point $\mathrm{d}$ passes through a normal shock and thus has a significantly higher entropy. However, far away from the leading edge, region $\mathrm{E}$ has a uniform pressure. The model further assumes that temperature and velocity are also uniform throughout region E. To calculate $T_{\mathrm{E}}$ and $u_{\mathrm{E}}$, consider the conservation of mass flux through the line connecting points a and $\mathrm{h}$. Then,

$$
\rho_{\mathrm{A}} u_{\mathrm{A}} \cos \theta_{\mathrm{A}}=\rho_{\mathrm{E}} u_{\mathrm{E}} \cos \theta_{\mathrm{E}}
$$

which can be used to iteratively solve for $T_{\mathrm{E}}$ assuming there is a relationship between $T_{\mathrm{E}}$ and $u_{\mathrm{E}}$. For an adiabatic nose,

$$
h_{\mathrm{A}}+\frac{1}{2} u_{\mathrm{A}}^{2}=h_{\mathrm{E}}+\frac{1}{2} u_{\mathrm{E}}^{2}
$$

\section{Simple Boundary-Layer Model}

The boundary-layer properties at the outflow of a scramjet inlet can have a significant impact on the performance of the combustion chamber. In addition, boundary layers tend to interact with and strengthen a shock near a leading edge. For these reasons an effort was made to include a model for a boundary layer. A displacement thickness is calculated, and the edge of the boundary layer is considered to be the actual surface of the vehicle. The displacement thickness, which is denoted $\delta$, is calculated using Eckert's reference temperature method. For a turbulent boundary layer, the reference temperature is

$$
T^{+}=\frac{1}{2}\left(T_{e}+T_{w}\right)+\sqrt[3]{\operatorname{Pr}^{+}}\left(T_{0}-T_{e}\right)
$$

where $T_{e}$ is the temperature at the edge of the boundary layer, and $T_{w}$ is the temperature of the wall. The edge conditions are taken from the entropy layer at a leading edge or whatever region is adjacent to the vertex in general.

To simplify the model further, the shape of the boundary layer is assumed to be a straight line connecting the current vertex and the edge of the boundary at the next downstream vertex. Consider a line that connects two vertices, a and b, of a polygon that represents an object in the flow. The total distance along the polygon boundary from the leading edge to the current vertex is $L_{\mathrm{a}}$, and the total distance from the leading edge to point $\mathrm{b}$ is $L_{\mathrm{b}}$. According to White [12], the change in displacement thickness for a turbulent boundary layer is approximately

$$
\delta_{\mathrm{b}}-\delta_{\mathrm{a}}=0.14 \frac{\eta^{+}}{\rho^{+} u_{e}}\left(L_{\mathrm{b}}^{6 / 7}-L_{\mathrm{a}}^{6 / 7}\right)
$$

Because the boundary-layer edge is considered to be a surface in this model, there is no flow across this surface. This means that the oblique wave must account for the increased area of the surface. Because the growth of the boundary layer is dependent on the conditions behind the shock or expansion, this creates a feedback between the strength of the wave in the inviscid region and the shape of the boundary layer. The current viscous model solves this system iteratively at each surface vertex that is not a trailing edge.

\section{Results}

For validation the proposed method is applied to a hypersonic inlet geometry that is a two-dimensional projection of an experimental geometry investigated by Emami et al. [13]. Experimental data were obtained for this configuration with a freestream Mach number of 4.0 for a range of cowl angles. Section IV.A compares these data to the results predicted by the two-dimensional reduced-order model.

The remaining results were obtained for an inlet geometry shown in Fig. 1, which has the dimensions listed in Table 1 . Figure 9 shows the results of the proposed method for the reference geometry at several flight conditions. For the reference inlet, the current implementation of the algorithm requires between 0.03 and $0.4 \mathrm{~s}$ assuming a calorically perfect gas. For the calorically imperfect gas model, the time required is between 0.8 and $6.5 \mathrm{~s}$ using Mathematica $^{\mathrm{TM}}$. The current MATLAB $\AA$ implementation requires about half the computational time, and further reductions can be expected using Fortran or $\mathrm{C}++$.

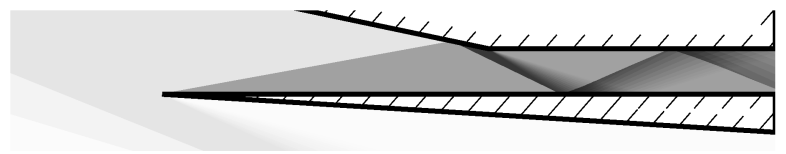

a) $M_{\infty}=6.0$

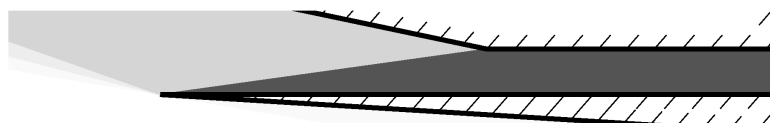

b) $M_{\infty}=8.0$

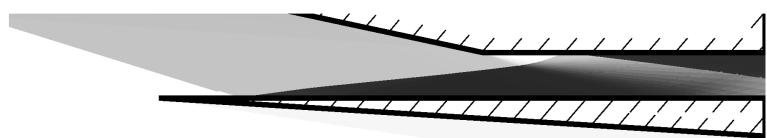

c) $M_{\infty}=10.0$

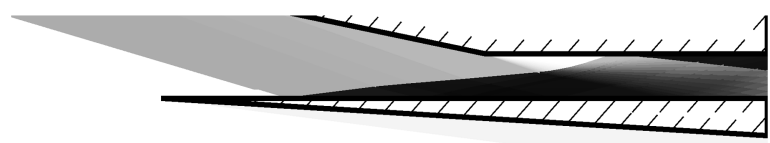

d) $M_{\infty}=12.0$

Fig. 9 Reduced-order model results for various flight freestream Mach numbers at an angle of attack of $\alpha=0$, where the darker shades represent regions of higher pressure, white represents freestream pressure, and black represents $p / p_{\infty}=90$. The expansion at the shoulder is modeled using $n_{\mathrm{ex}}=20$ in each case. 


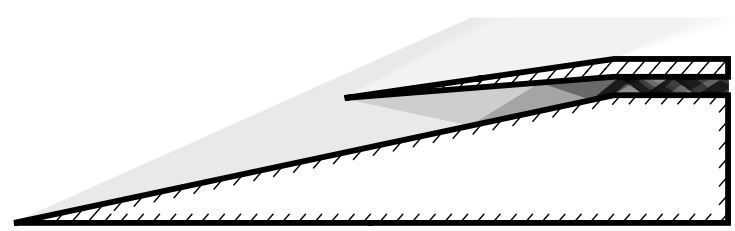

Fig. 10 Reduced-order solution for the Emami et al. [13] geometry with $\theta_{\mathrm{cl}}=6.5 \mathrm{deg}$, where the darker shades represent regions of higher pressure, white represents freestream pressure, and black represents $p / p_{\infty}=40$.

\section{A. Comparison to Experiment}

A sample geometry of the experiment of Emami et al. [13] is shown in Fig. 10. The inlet has the opposite vertical orientation of the inlet in Fig. 1 to be consistent with the experimental apparatus and keep it clearly separate from the three-ramp inlet used elsewhere in the paper.

The relevant dimensions of the inlet are shown in Table 2 . In Fig. 10, the angle between horizontal and the bottom surface of the cowl leading edge is denoted $\theta_{\mathrm{cl}}$. The hinge for this rotation is directly above (has the same $x$ coordinate) as the inlet shoulder. In the experimental data, $\theta_{\mathrm{cl}}$ was allowed to vary between 0 and $11 \mathrm{deg}$. However, small values of $\theta_{\mathrm{cl}}$ cause the inlet to unstart.

The experiment of Emami et al. [13] had pressure taps, which can measure static pressure along a surface at many points along the centerline of the geometry. Three pressure taps downstream of the cowl leading edge are used to compare the experimental results with the reduced-order model results. The first pressure tap is along the bottom surface of the cowl $5.84 \mathrm{~cm}$ downstream of the cowl leading edge. The second pressure tap is on the bottom surface of the cowl $10.3 \mathrm{~cm}$ downstream of the cowl leading edge. The third pressure tap is on the inlet ramp $0.23 \mathrm{~cm}$ upstream of the shoulder. These pressures were recorded for a range of $\theta_{\mathrm{cl}}$. For comparison, the pressures were calculated at the same points using the sharp-edged viscous version of the proposed reduced-order model. The combined results are shown in Fig. 11.

The internal section of the experimental inlet was only $5.08 \mathrm{~cm}$ wide, meaning that the aspect ratio of the isolator was 5.0. Although higher aspect ratios would better justify the assumption of a twodimensional flow (which is explicit in the proposed reduced-order model), the predicted results are quite similar to the experimental data. There is a $10 \%$ underprediction of the pressure at station 1 , which is likely due to a pressure increase caused by a bow shock at the very slightly rounded cowl leading edge. This is also a possible cause of the underpredicted pressures at the other two stations for low values of $\theta_{\mathrm{cl}}$. There is also a disagreement about shock positions. The evidence of a shock can be readily seen at stations 1 and 2 in both the experimental and modeled data sets. However, the two data sets disagree about the value of $\theta_{\mathrm{cl}}$ that causes the shock to pass over each station. This is most likely due to unmodeled shock/boundary-layer interactions. The present reduced-order viscous model only models shock/boundary-layer interactions at leading edges and other corners of the inlet surface, and Fig. 10 clearly shows that the cowl shock interacts with the boundary layer of the inlet ramp upstream of the inlet shoulder. As a result, the present reduced-order model underpredicts the angle of the reflected shock by about $1 \mathrm{deg}$.

At other locations, however, the predicted pressure matches closely. Except for the incorrect shock position in state 1, all errors are bounded to $10 \%$. The remaining errors could be reduced by using the model for a blunted leading edge. The edges were assumed to be

Table 2 Relevant lengths for the experimental inlet of Emami et al. [13]; the inlet ramp has an $11 \mathrm{deg}$ incline

\begin{tabular}{lc}
\hline \hline Surface & Length \\
\hline Inlet ramp (horizontal) & $24.816 \mathrm{~cm}$ \\
Inlet ramp (vertical) & $4.8260 \mathrm{~cm}$ \\
Isolator height & $1.0160 \mathrm{~cm}$ \\
Forward cowl length & $11.176 \mathrm{~cm}$ \\
\hline \hline
\end{tabular}

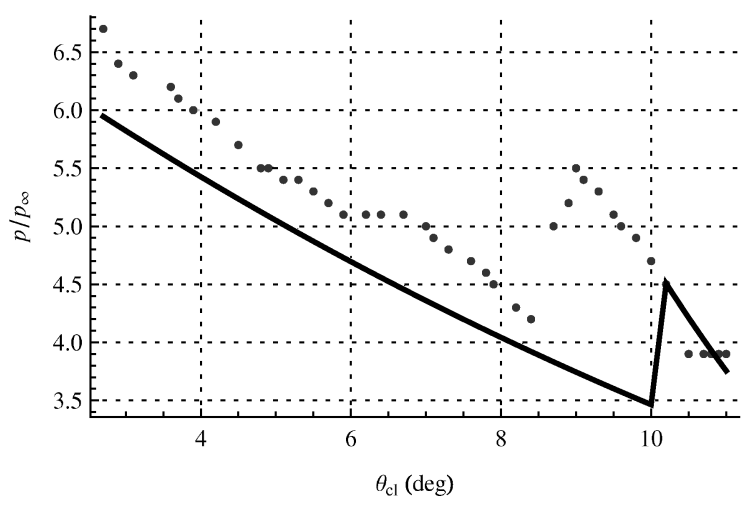

a) Station 1

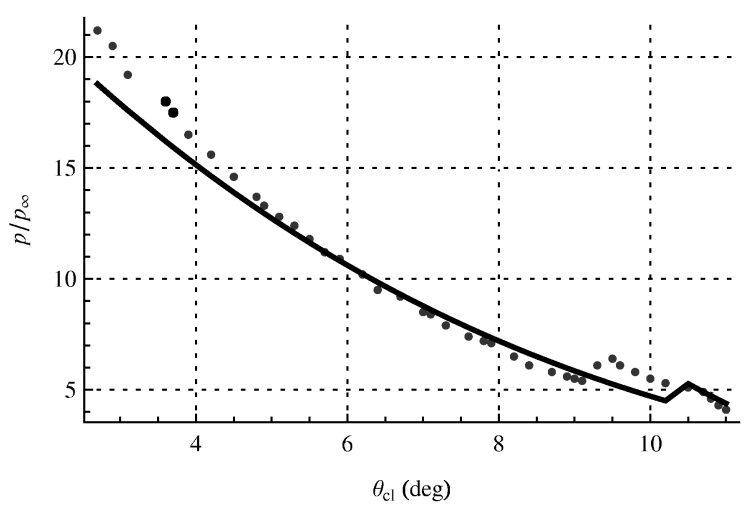

b) Station 2

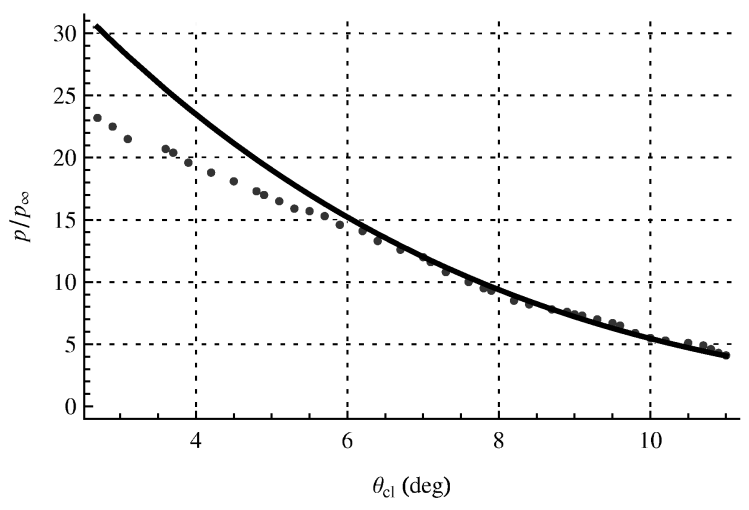

c) Station 3

Fig. 11 Comparison of experiment and reduced-order model. The dots are data from Emami et al. [13], and the solid line shows the prediction of the reduced-order model.

infinitely sharp in this comparison because no data for the edge radius of the experimental setup were provided. This provides a measure of experimental validation for the proposed method. A comparison to experimental values of $p_{2} / p_{\infty}$ and $p_{02} / p_{0 \infty}$, as defined in Sec. III.B, would be better, but that would be difficult data to obtain experimentally.

\section{B. Comparison to CFD}

CFD was used to compute the viscous flow over the reference inlet at a flight Mach number of $M_{\infty}=10$ and an angle of attack of $\alpha=0$. The reference inlet was recreated by a two-dimensional grid consisting of approximately $1.3 \times 10^{6}$ triangular cells. This grid was constructed such that special attention was given to capturing the correct boundary-layer effects along the length of the inlet ramps, as well as providing for resolving appropriate oblique shock thicknesses inside the engine intake. 


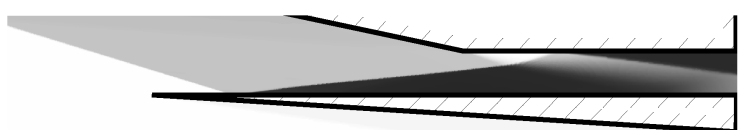

Fig. 12 High-fidelity viscous flow solution as computed by CFD++. The flight Mach number is $M_{\infty}=10$, and the angle of attack is $\alpha=0$. The color scale is the same as Fig. 9.

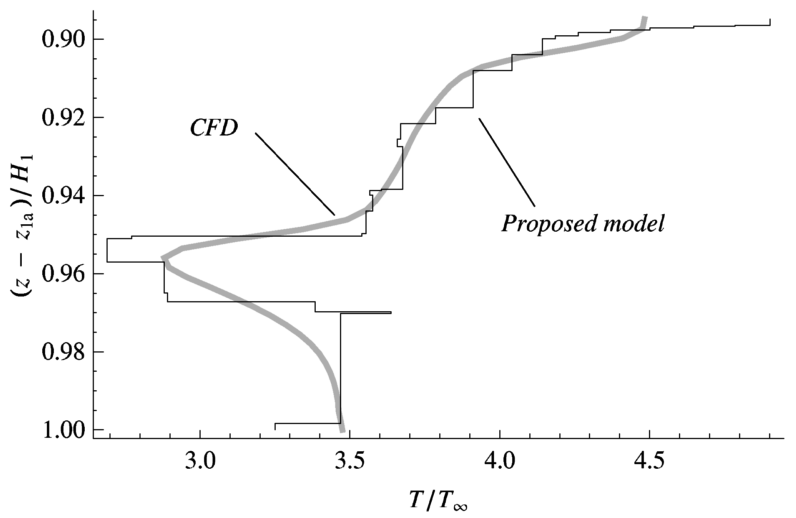

Fig. 13 Normalized temperature along the $z$ axis of the inlet outflow plane for both the calorically imperfect inviscid inlet model and a twodimensional CFD result.

The steady viscous simulation of the inlet was created using the commercial CFD package CFD++, operating across a parallel cluster of 8 AMD 64-bit Opteron processors, each with a minimum of 2 GB of RAM. This arrangement allowed for a total simulation time of approximately four days, consisting of just over 30,000 solver iterations, in which a normalized convergence on the order of $10^{-4}$ was reached. The turbulence in the flow was modeled using a standard $k-\varepsilon$ scheme.

The results of this CFD computation are shown in Fig. 12. The CFD result appears very similar to that of Fig. 9c, which is the reduced-order simulation of the same flight conditions. As a quantification of this similarity, Fig. 13 shows the nondimensional temperature along the downstream boundary of the inlet. Each vertical segment of the result from the proposed inviscid calorically imperfect model represents the temperature of one polygon of the flow solution. The two results have the same trend, and the average temperature, $T_{2}$, is accurate to within $1.16 \%$. The spatially averaged properties from both solution methods are listed in Table 3. This comparison shows that the calorically imperfect inviscid model can be expected to predict the thermodynamic properties to within $10 \%$.

\section{Resolution of Discrete Expansion Fans}

One of the key parameters directly affecting the amount of computation time required in this model is the number of discrete waves in an expansion fan, $n_{\mathrm{ex}}$. Therefore, determining an appropriate value of $n_{\mathrm{ex}}$ is crucial for maximizing the efficiency of the code. Figure 14 shows the relative error associated with using small values of $n_{\mathrm{ex}}$ for one flight condition of the reference inlet. The three quantities considered are the pressure recovery factor, $p_{0,2} / p_{0, \infty}$; the compression ratio, $p_{2} / p_{\infty}$; and the average outflow Mach number, $M_{2}$. The large errors that occur when an expansion is modeled as a single wave can be explained by the fact that single-wave expansions

Table 3 Averaged outflow properties of CFD and calorically imperfect inviscid model

\begin{tabular}{lccc}
\hline \hline & CFD & Proposed model & Relative error \\
\hline$p_{2} / p_{\infty}$ & 34.70 & 35.39 & 0.0199 \\
$T_{2} / T_{\infty}$ & 3.603 & 3.645 & 0.0156 \\
$u_{2} / u_{\infty}$ & 0.9289 & 0.9302 & 0.0013 \\
$p_{0,2} / p_{0, \infty}$ & 0.3331 & 0.3552 & 0.0664 \\
\hline \hline
\end{tabular}

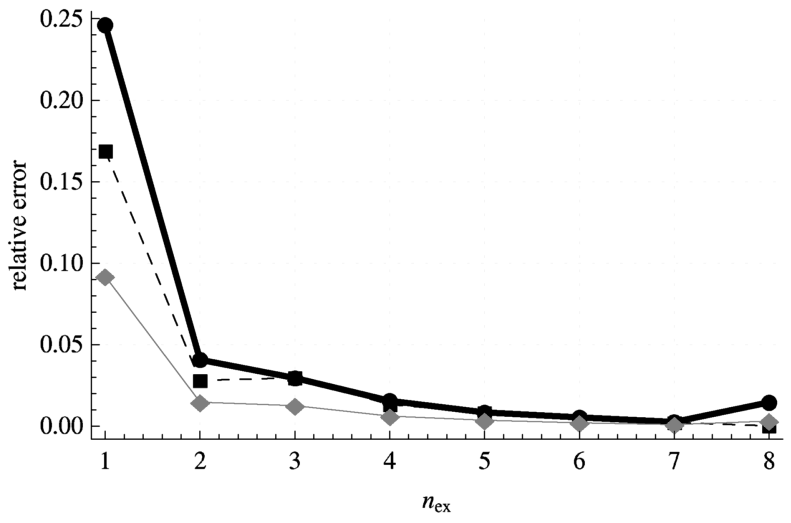

Fig. 14 Relative errors in $p_{0,2} / p_{0, \infty}$ (bold line with circles), $p_{2} / p_{\infty}$ (dashed line with squares), and $M_{2}$ (gray line with diamonds) plotted against the expansion discretization parameter, $n_{\mathrm{ex}}$, for the reference inlet at a flight condition of $M_{\infty}=10.0$ and $\alpha=0$. The reference values were obtained using $n_{\mathrm{ex}}=\mathbf{4 0}$.

do not occupy any area, and as a result, the locations of the wave interactions are not correct.

However, for any value of $n_{\mathrm{ex}}$ other than one, the results are quite similar. Errors of less than 5\% could likely be considered acceptable considering that the error associated with assuming a twodimensional flow is at least as large. Therefore, the results of the following sections are obtained using $n_{\mathrm{ex}}=2$.

\section{Effects of Varying Flight Conditions on Reference Inlet}

One of the most important benefits of this solution method is the ability to estimate the performance of a scramjet inlet over a wide

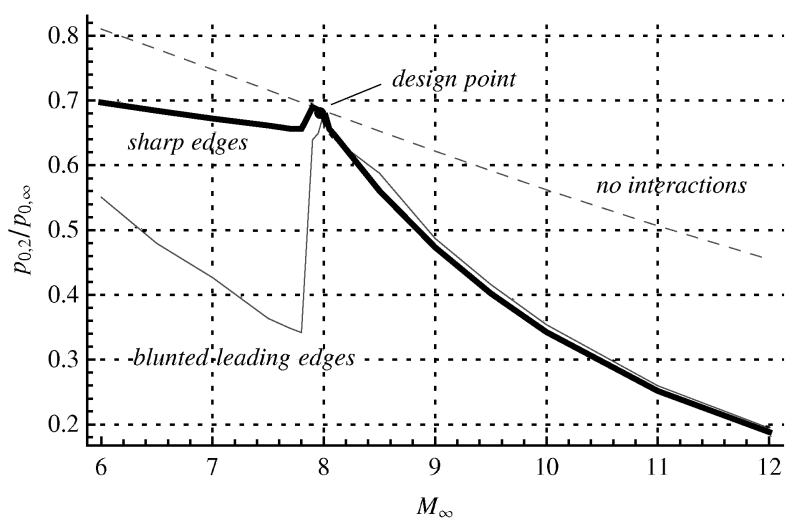

a) $\alpha=0$

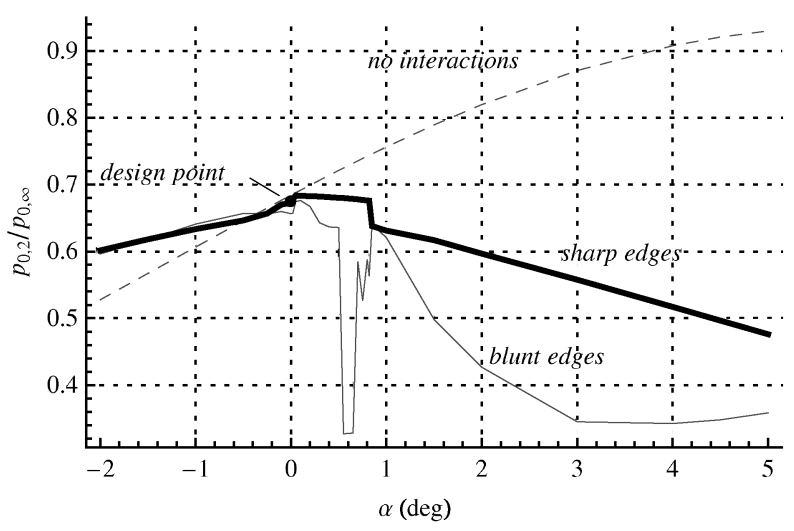

b) $M_{\infty}=8.0$

Fig. 15 Pressure recovery factor, $p_{0,2} / p_{0, \infty}$, plotted for the reference inlet at various flight conditions. The design flight condition of $M_{\infty}=8.0$ and $\alpha=0$ is marked. 


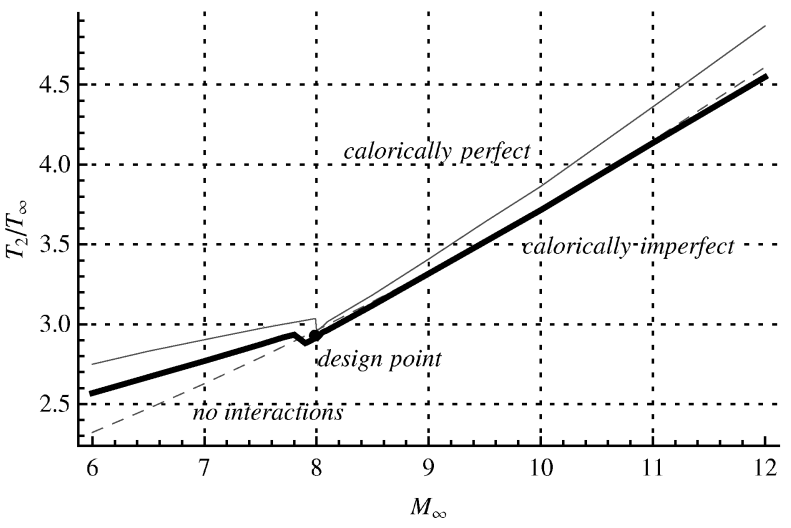

a) $\alpha=\mathbf{0}$

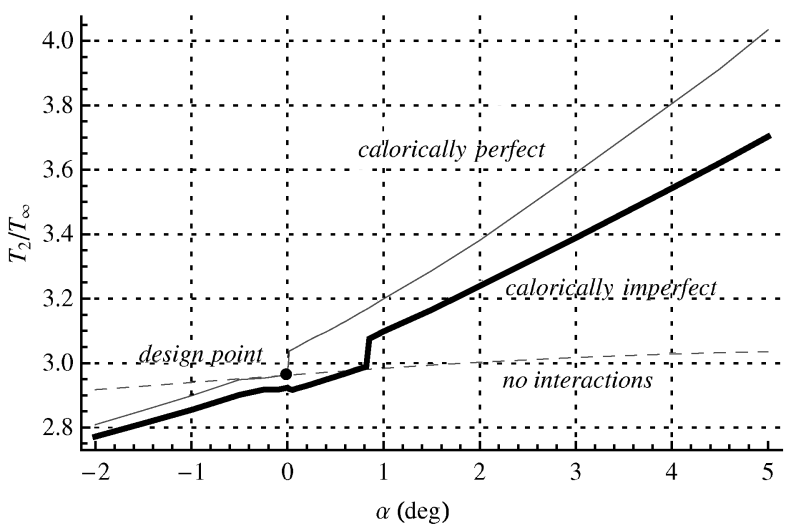

b) $M_{\infty}=8.0$

Fig. 16 Averaged inlet outflow temperature plotted for the reference at various flight conditions. The design flight condition of $M_{\infty}=8.0$ and $\alpha=0$ is marked.

range of flight conditions. Figures 15 and $\underline{16}$ show plots of the pressure recovery factor and $T_{2}$, respectively, for the reference inlet assuming either a constant angle of attack or constant freestream Mach number. For the cases requiring blunted leading edges, nose radii of $r_{\mathrm{le}}=5 \mathrm{~mm}$ are assumed. For comparison, the results of a further simplified model are shown as a dotted line in Figs. 15 and 16. In this model there are only four shocks, which are assumed to have the locations shown Fig. 1 regardless of flight conditions. In this model there are no shock interactions, and the inlet outflow is always uniform.

For the calorically perfect gas model, the flow can be parameterized entirely by $M_{\infty}$ and $\alpha$. On the other hand, the thermally perfect gas model also requires a stagnation enthalpy. To determine $h_{0}$, a constant dynamic pressure of $97.8 \mathrm{kPa}$ is assumed. The dynamic pressure and Mach number uniquely define a static pressure. With the freestream pressure known, the freestream temperature is determined using a standard atmosphere model.

Figure 15, which shows the effects of real gas considerations and wave interactions on the pressure recovery factor for the reference inlet, shows that the simplified model predicts performance accurately near the design condition of $M_{\infty}=8.0, \alpha=0$. At higher Mach numbers, the more realistic pressure recovery factor estimate is much lower, that is, both the solid lines in Fig. 15 lie well below the dotted line except at the design point. This can be explained by the strong shocks that continue to reflect in the internal portion of the inlet. For lower angles of attack, the simplified model actually predicts a lower pressure recovery factor. This is because of the expansion at the inlet shoulder, which is not considered in the simplified model. Thus, the increased pressure recovery factor is obtained at the cost of a lower static pressure.

For lower flight Mach numbers, the blunted leading edge has a very significant effect. This happens because the blunted leading edge of the cowl pushes the interaction of the cowl shock even further

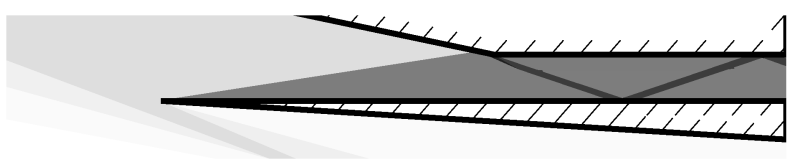

a) Sharp leading edges

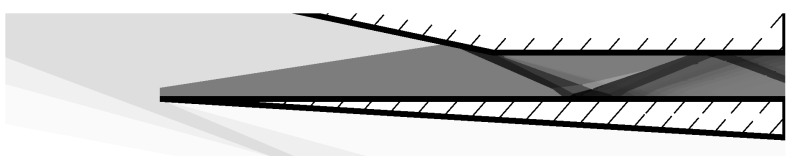

b) Blunted leading edges

Fig. 17 Inviscid flow for the internal portion of the inlet for a flight condition of $M_{\infty}=7.0$ and $\alpha=0$, where the darker shades represent regions of higher pressure, white represents freestream pressure, and black represents $p / p_{\infty}=90$. The expansion at the shoulder is modeled using $n_{\mathrm{ex}}=2$ in each case.

upstream of the shoulder, as shown in Fig. 17. Because the cowl shock has moved upstream, it does not interact with the expansion at the shoulder before reflecting off the inlet body. Similar effects occur when the angle of attack is increased above the design condition, resulting in sharp discontinuity in the pressure recovery factor near $\alpha=0.6 \mathrm{deg}$. An increasing angle of attack has an effect similar to a decreasing Mach number because the initial bow shock must turn the flow through a greater angle, which increases the strength of the shock. Thus, if the preshock Mach number is held constant, the postshock Mach number will decrease as the angle of attack increases.

The predictions of the calorically perfect and calorically imperfect gas models are compared in Fig. 16. For a given enthalpy, the calorically imperfect model will predict a lower temperature. Thus, it is possible for the temperature estimate to be significantly changed by the choice of the gas model while the pressure and density are relatively unaffected. In addition, the wave angles will be slightly different for each model, and so the conditions at which the cowl shock moves upstream of the shoulder will be changed. This explains the differing locations of the discontinuities in Fig. 16.

\section{Conclusions}

The present work shows that it is possible to use the proposed reduced-order model to compute the spatially averaged properties on the output plane of a supersonic inlet to an accuracy of $3 \%$ with a computational time of less than a few seconds. The proposed method includes the effects of multiple wave interactions and a calorically imperfect gas model necessary for the high temperatures in hypersonic flows. The results were compared to experimental data, and the model inaccuracies were found to be limited to $10 \%$. Furthermore, possible improvements were made visible. An architecture is also introduced that allows for the modeling of blunted leading edges.

The computational savings relative to CFD are immense, and there is also no need to assemble a grid beforehand. The method can be applied to a geometry under a wide range of supersonic conditions. For a control-oriented model, these properties are essential. Scramjet inlets are a particularly useful application of this algorithm. A control-oriented model of the scramjet isolator, combustor, and nozzle requires an accurate estimate of the thermodynamic properties at the downstream boundary of the inlet. A comparison to CFD shows that the averaged thermodynamic properties at the end of a scramjet inlet can be estimated, and the proposed model can be used in a control-oriented model of a scramjet vehicle.

\section{Acknowledgments}

This research was supported by U.S. Air Force Research Laboratory grant FA 8650-07-2-3744 for the Michigan Air Force Research Laboratory Collaborative Center for Control Science and by NASA grant NNX08AB32A, administered by Donald Soloway, technical monitor. 


\section{References}

[1] Bogar, T. J., Alberico, J. F., Johnson, D. B., Espinosa, A. M., and Lockwood, M. K., "Dual-Fuel Lifting Body Configuration Development," AIAA Paper 96-4592, 1996.

[2] Bolender, M. A., and Doman, D. B., "Nonlinear Longitudinal Dynamical Model of an Air-Breathing Hypersonic Vehicle," Journal of Spacecraft and Rockets, Vol. 44, No. 2, 2007, pp. 374-387. doi: $10.2514 / 1.23370$

[3] Brown, M., Mudford, N. R., Neely, A. J., and Ray, T., "Robust Optimization of Two-Dimensional Scramjet Inlets," AIAA Paper 20068140, 2006.

[4] Chavez, F. R., and Schmidt, D. K., "Analytical Aeropropulsive/ Aeroelastic Hypersonic-Vehicle Model with Dynamic Analysis," Journal of Guidance, Control, and Dynamics, Vol. 17, No. 6, 1994, pp. 1308-1319. doi:10.2514/3.21349

[5] Smart, M. K., "Optimization of Two-Dimensional Scramjet Inlets," Journal of Aircraft, Vol. 36, No. 2, 1999, pp. 430-433. doi: $10.2514 / 2.2448$

[6] Billig, F. S., "Shock-Wave Shapes Around Spherical- and CylindricalNosed Bodies," Journal of Spacecraft and Rockets, Vol. 4, 1967, pp. 822-823. doi: $10.2514 / 3.28969$

[7] Emanuel, G., Analytical Fluid Dynamics, CRC Press, Boca Raton, FL, 2001, Chap. 9.

[8] Ismail, M., "Prandtl-Meyer Flow of a Calorically Imperfect Gas," Master's Thesis, School of Aerospace and Mechanical Engineering, Univ. of Oklahoma, Norman, OK, 1994.

[9] Zebbiche, T., "Stagnation Temperature Effect on the Prandtl-Meyer Function," AIAA Journal, Vol. 45, No. 4, 2007, pp. 952-954. doi: $10.2514 / 1.24868$

[10] Tatum, K. E., "Computation of Thermally Perfect Oblique Shock Wave Properties," AIAA Paper 1997-868, 1997.

[11] Anderson, J. D., Fundamentals of Aerodynamics, 2nd ed., McGrawHill, New York, 1991.

[12] White, F. M., Viscous Fluid Flow, 3rd ed., McGraw-Hill, New York, 2006.

[13] Emami, S., Trexler, C. A., Auslender, A. H., and Weidner, J. P., "Experimental Investigation of Inlet-Combustor Isolators for a DualMode Scramjet at a Mach Number of 4," NASA Technical Paper 3502, May 1995.

R. Bowersox Associate Editor 